\title{
A cross-sectional study of susceptibility to vaccine-preventable diseases among prison entrants in New South Wales
}

\begin{abstract}
Sarah Larney BA, BSc(Hons), PhD, NHMRC Early Caree Research Fellow, National Drug and Alcohol Research Centre,' and Senior Research Officer, Centre for Health Research in Criminal Justice ${ }^{2}$

Denise L Monkley BNSC(Hons), MPH, Service Director, Population Health, Clinical and Nursing Services

Devon Indig PhD, MPH, BSC, Head of Research, Centre for Health Research in Criminal Justice, ${ }^{2}$ and

Conjoint Senior Lecturer School of Public Health and Community Medicine

Stephen E Hampton MB BS, MPH, FRACGP Clinical Director

1 University of New South Wales, Sydney, NSW

2 Justice Health and Forensic Mental Health Network, Sydney, NSW

s.larney@unsw.edu.au
\end{abstract}

MJA 2013; 198: 376-379 doi:10.5694/mjal2.11ו10
T': he custodial environment poses challenges for infectious disease management. ${ }^{1}$

With close contact between inmates and high population turnover, infectious diseases can spread rapidly. Prison outbreaks of vaccine-preventable diseases including mumps, ${ }^{2}$ varicella $^{3,4}$ and hepatitis $B^{5,6}$ have been reported.

Health care services in New South Wales prisons are provided by Justice Health and Forensic Mental Health Network, a statutory health corporation under the Ministry of Health. In August 2010, a measles outbreak was detected in prisons on the NSW North Coast. After this outbreak was contained, it was determined that more information was needed about susceptibility to vaccine-preventable diseases among adults in custody, to inform vaccination policies and clinical practices. At present, there is no policy on vaccination of prisoners against varicella, measles, mumps or rubella in NSW prisons. It is policy that all inmates be offered hepatitis B vaccination, ${ }^{7}$ but it is unclear what proportion of inmates commence or complete the vaccination schedule.

In this study, we aimed to determine the prevalence of susceptibility to measles, mumps, rubella, varicella and hepatitis B virus (HBV) among people entering prisons in NSW. Our secondary aims were to identify sociodemographic characteristics associated with disease susceptibility and to compare the susceptibility of prison entrants with the susceptibility of a community sample.

\section{Methods}

This study was undertaken as a NSWonly add-on to the triennial National Prison Entrants' Bloodborne Virus and Risk Behaviour Survey. ${ }^{8}$ Both studies were approved by the Justice Health Human Research and Ethics Committee.

\begin{abstract}
Objectives: To determine the prevalence of susceptibility to measles, mumps, rubella, varicella and hepatitis B virus (HBV) among New South Wales prison entrants and to compare results for prison entrants with those of a community sample.
\end{abstract}

Design and setting: Between 11 October 2010 and 24 October 2010, new entrants at seven adult correctional centres completed a cross-sectional survey and provided a venous blood sample.

Participants: All adults entering the correctional centres were eligible to participate, with 211 completing the survey (response rate 68\%).

Main outcome measures: Serological evidence of immunity to measles, mumps, rubella, varicella and HBV. Prison data were compared with community data obtained from the 2007 Australian National Serosurveillance Program.

Results: Over half of the participants $(106 / 204,52 \%)$ were susceptible to HBV, followed by susceptibility to mumps $(82 / 198,41 \%)$, rubella $(33 / 209,16 \%)$, measles $(27 / 203,13 \%)$ and varicella $(19 / 198,10 \%)$. Having no history of drug injection was a significant predictor of susceptibility to measles, mumps and HBV. Prison entrants were significantly less likely than people in the community to be susceptible to varicella (10\% versus 18\%; risk ratio [RR], 1.9; 95\% Cl, 1.13.2 ) and HBV (52\% versus 65\%; RR, 1.3; 95\% Cl, 1.1-1.5).

Conclusions: Prison entrants are susceptible to a number of vaccinepreventable diseases. We recommend a cost-benefit analysis of implementing routine vaccination for measles, mumps, rubella and varicella and an exploration of options for improving uptake of HBV vaccination.

\section{Recruitment}

Between 11 October 2010 and 24 October 2010, participants were recruited in all seven NSW prison reception centres operating at the time of the survey. In 2010, the average daily number of new entrants was 29. All individuals entering prison from the community who were able to provide informed consent were eligible to participate. We were unable to exclude entrants who may have been vaccinated against measles, mumps and rubella as part of the response to the earlier measles outbreak. New prisoners were called to the clinic within 24 hours of entry into the prison and provided with an explanation of the project by a member of the interviewing team. Written informed consent was obtained from all participants. Blood test results were returned to participants and vaccination was offered where appropriate.

\section{Data collection}

All interviewers were trained in administering the questionnaire and accredited in venepuncture. Questionnaire items were related to sociodemographics, prior incarcerations, injecting drug use (IDU) and other risk behaviours. To protect participant confidentiality, questionnaires and venous blood samples were labelled using a coded identifier.

Blood samples were analysed at the Institute of Clinical Pathology and Medical Research, Westmead Hospital. Some samples were of insufficient volume to be tested for all target infections, resulting in varying sample sizes for each infection. Measles, mumps and varicella zoster virus-specific IgG antibody titres were measured using ELISA (enzyme-linked immunosorbent assay) kits (Enzygnost). Rubella IgG antibody titres were measured using microparticle enzyme immunoassays (Architect; Abbott Diagnostics). Samples were classed as antibodypositive, antibody-negative or equivocal in accordance with the test manufacturer's standards. Participants with antibody-negative or 
equivocal results were considered susceptible to infection.

Hepatitis B core antibody (anti$\mathrm{HBc})$, hepatitis $\mathrm{B}$ surface antibody (anti-HBs) and hepatitis B surface antigen (HBsAg) were measured using microparticle enzyme immunoassays (Architect; Abbott Diagnostics). Participant results were classified as follows:

- participants with results that were negative to both anti-HBc and HBsAg were considered susceptible to HBV infection;

- participants with results that were positive to both anti-HBc and HBsAg were considered to have a current infection;

- participants with results that were positive for anti-HBC with an antiHBs level of $\geqslant 10 \mathrm{mIU} / \mathrm{mL}$ but negative to HBsAg were considered immune as a result of past infection; and

- participants with an anti-HBs level of $\geqslant 10 \mathrm{mIU} / \mathrm{mL}$ and results that were negative to both anti-HBc and HBsAg were considered immune as a result of vaccination.

\section{Comparison data}

The prison data were compared with NSW data from the 2007 Australian National Serosurveillance Program (ANSP). The ANSP collects stored serum samples from public and private diagnostic laboratories in each Australian state and territory. The collected sera are tested for antibodies to a variety of infectious diseases, including those tested in the Vaccine Preventable Diseases Study (VPDS). ${ }^{9,10}$ The data provided by the ANSP were the number of individuals, broken down by sex and birth cohort, with positive, equivocal and negative results for measles, mumps, rubella and varicella antibodies, and serological markers of HBV. Susceptibility to each disease was defined in the same manner as the prison data.

\section{Data analysis}

Data were analysed using SAS version 9.3 (SAS). Susceptibility to each disease was calculated and the $\chi^{2}$ test was used to test for differences according to sociodemographic characteristics and risk behaviours. For each disease, variables with $P \leqslant 0.05$ $\left(\chi^{2}\right.$ test $)$ were entered into logistic regression models to determine independent predictors of susceptibility

The ANSP data were weighted to match the birth cohort and sex distribution of the prison sample. Immunity of the prison and community samples to each disease was compared by calculating weighted risk ratios and $95 \%$ confidence intervals.

\section{Results}

Of 311 prison entrants approached to participate in the study, 211 (68\%) completed the questionnaire and provided a blood sample. Participants were similar to non-participants in terms of Aboriginality and age. Women disproportionately declined to participate, comprising $3 \%$ of participants and $19 \%$ of non-participants.

Of the participant sample, 97\% (204/211) were male, and 21\% (44/ 211) identified as Aboriginal or Torres Strait Islander. The median age was 32 years (range, 17-79 years). Almost two-thirds of the participants $(65 \%$; $138 / 211$ ) had previously been in prison, and 37\% (75/202) had ever injected drugs (Box 1).

\section{Measles, mumps, rubella and varicella}

Of 203 participants with measles serology, 13\% (27/203) were susceptible to infection (Box 1). Susceptibility was significantly higher in younger birth cohorts $(P=0.04)$, those with lower education levels $(P=0.02)$, and participants who had never injected drugs $(P=0.03)$. In multivariate analysis, birth cohort was not significantly associated with measles susceptibility $(P=0.1)$; however, less education $(P=$ $0.02)$ and no history of IDU $(P=0.05)$ remained significant.

Of 198 participants with mumps serology, 41\% (82/198) were susceptible to infection. Susceptibility was significantly higher among people born in Australia $(P=0.01)$, those with lower education levels $(P=0.05)$, and those without a history of IDU $(P=$ 0.02). In multivariate analysis, being Australian-born $(P=0.009)$ and having no history of IDU $(P=0.007)$ remained significant predictors of mumps susceptibility.

Sixteen per cent of the participants $(33 / 209)$ were susceptible to rubella, and 10\% (19/198) were susceptible to varicella. There were no significant associations between susceptibility to rubella or varicella and demographic or behavioural characteristics.

\section{Hepatitis B}

Just over half of participants (52\%; $106 / 204$ ) were susceptible to HBV infection (Box 1); 3\% (6/204) had acute or chronic infection, and $45 \%$ (92/204) were immune. Susceptibility was significantly higher among participants who were entering prison for the first time $(P=0.005)$, and those with no history of IDU $(P=0.003)$. In multivariate analysis, no history of IDU remained a significant predictor of susceptibility to $\mathrm{HBV}$ infection $(P=0.03)$.

Among the 92 participants who were HBV immune, 36\% (33/92; 16\% of the total sample) had acquired immunity through prior infection, and $64 \%$ (59/92; $29 \%$ of the total sample) had vaccine-conferred immunity. Post-hoc sub-analyses were undertaken to identify correlates of vaccineconferred immunity among those with HBV data. Participants with a history of IDU were significantly more likely than than those with no history of IDU to have been vaccinated, with $28 / 72(39 \%)$ of those with a history of IDU having been vaccinated, versus $31 / 126(25 \%)$ of those with no history of IDU $(P=0.03)$. There was no significant relationship between prior incarceration and vaccine-conferred immunity; 44/134 participants (33\%) who had previously been incarcerated had been vaccinated, compared with $15 / 70$ (21\%) who were entering prison for the first time $(P=0.08)$. There was no significant relationship between prior incarceration and vaccine-conferred immunity when the analysis was restricted to participants with a history of IDU; $27 / 63$ (43\%) of IDU prisoners with a prior incarceration were vaccinated, compared to $1 / 9$ $(11 \%)$ of IDU prisoners entering prison for the first time $(P=0.07)$.

\section{Community comparison}

Data from the ANSP were weighted and compared with the prison entrants' data. Prison entrants were significantly less likely than those in the general community to be susceptible to varicella ( $10 \%$ versus $18 \%$; $P=0.01)$ and HBV (52\% versus $65 \%$; 
1 Demographic and behavioural characteristics and susceptibility to measles, mumps, rubella, varicella and hepatitis B virus among 211 prison entrants in New South Wales

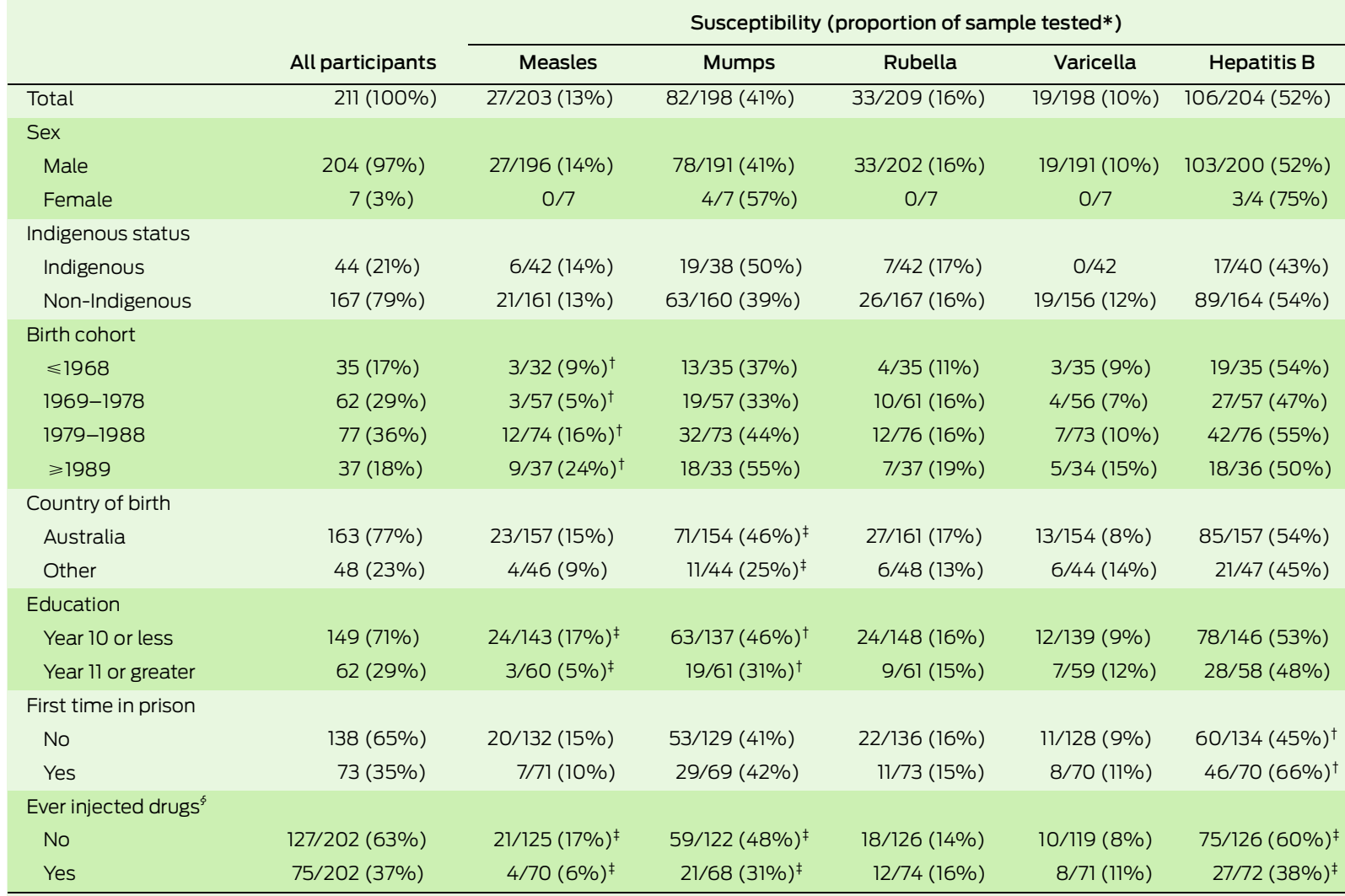

* Sample size varies with each disease because some samples were of insufficient volume to be tested for all target infections. $\dagger P \leqslant 0.05$ using $\chi^{2}$ test. $\ddagger P \leqslant 0.05$ in multivariate logistic regression models with participant characteristics as independent variables. $\oint$ Data were missing for nine participants.

$P=0.007)$. There were no significant differences between the prison and community samples in terms of susceptibility to measles, mumps or rubella (Box 2).

\section{Discussion}

Our analysis shows that the proportion of NSW prison entrants who are susceptible to vaccine-preventable diseases varies widely with each disease. Although prisoners' susceptibility was lower than that of the general community for some diseases, recent experience with measles in NSW has shown that there are sufficient numbers of susceptible prisoners for outbreaks to occur. Recent work suggests that vaccination coverage of more than $95 \%$ may be necessary for the prevention of measles outbreaks. ${ }^{11}$ Entry into custody is an opportune time to routinely offer vaccinations against these infectious diseases in order to ensure high levels of immunity across the prisoner population.
Combination measles-mumpsrubella vaccine, varicella vaccine, and $\mathrm{HBV}$ vaccine are all well tolerated by individuals who have previously been infected or vaccinated; as such, all individuals with an uncertain infection and vaccination history and without contraindications can safely commence these vaccination schedules. To our knowledge, the costs and benefits of routine vaccination in correctional settings have not been evaluated - a cost-benefit analysis would be an appropriate next step in developing vaccination policies.

Birth cohort and Aboriginality were not significant predictors of susceptibility to our target diseases. Participants born outside Australia were less likely to be susceptible to mumps, likely reflecting greater exposure to wild virus in the country of birth. No history of IDU was significantly associated with susceptibility to measles, mumps and HBV. In the case of measles and mumps, it is not clear if this association is a result of higher vacci- nation rates or exposure to wild virus. For HBV, there was some evidence of higher levels of vaccination among inmates with a history of IDU compared with those without. Despite policies mandating that $\mathrm{HBV}$ vaccine be offered to all inmates, there was no association between prior incarceration and $\mathrm{HBV}$ vaccination. A clear opportunity exists to improve HBV vaccination coverage among NSW prisoners in general, particularly those who inject drugs. The largest increases in $\mathrm{HBV}$ vaccination coverage are obtained through routine vaccination of prison entrants rather than in repeated mass campaigns. ${ }^{12,13}$ Administering vaccine via an accelerated schedule (on the day of entry into prison, 7 days after entry, 21 days after entry and a 12-month booster) can increase the proportion of prisoners completing the vaccination schedule. ${ }^{14}$

\section{Limitations}

This study included participants from all reception centres and we achieved 
2 Comparison of susceptibility to measles, mumps, rubella, varicella and hepatitis $B$ virus in the community and in 211 prison entrants in New South Wales

Proportion susceptible

\begin{tabular}{lccc} 
& \multicolumn{2}{c}{} & \\
\cline { 2 - 3 } & $\begin{array}{c}\text { Community sample } \\
\text { (weighted percentage) } *^{\dagger \dagger}\end{array}$ & Prison entrants & Risk ratio ${ }^{\ddagger}(95 \% \mathrm{Cl})$ \\
\hline Measles & $217 / 1281(16 \%)$ & $27 / 203(13 \%)$ & $1.2(0.7-2.2)$ \\
Mumps & $579 / 1281(44 \%)$ & $82 / 198(41 \%)$ & $1.1(0.8-1.3)$ \\
Rubella & $117 / 1281(15 \%)$ & $33 / 209(16 \%)$ & $0.9(0.6-1.5)$ \\
Varicella & $394 / 1229(18 \%)$ & $19 / 198(10 \%)$ & $1.9(1.1-3.2)^{5}$ \\
Hepatitis B & $602 / 933(65 \%)$ & $106 / 204(52 \%)$ & $1.3(1.1-1.5)^{5}$ \\
\hline
\end{tabular}

*Actual number of participants; percentage weighted to match the birth cohort and sex distribution of the prison sample. $†$ Data from the 2007 Australian National Serosurveillance Program. $¥$ Risk ratio is the ratio of weighted risk in the community sample divided by the risk in the prison sample. $\S P \leqslant 0.05$ using the $\chi^{2}$ test.

a moderate response rate $(68 \%)$. Some entrants with very short stays in prison (eg, less than 24 hours) may not have been approached to participate because of staffing limitations; we were unable to determine what proportion of entrants were not approached to participate. Participants were highly representative of the prisoner population in terms of age (participant median age, 32 years, versus population median age, 30-34 years) and Aboriginality ( $21 \%$ of participants versus $22 \%$ of population), ${ }^{15}$ but women were underrepresented in our sample, such that we were unable to draw conclusions about differences in susceptibility according to sex.

\section{Conclusions}

Currently, the Australian immunisation handbook recommends influenza, hepatitis A and hepatitis B vaccinations for prisoners. ${ }^{16}$ Given the potential for respiratory-spread infectious diseases to spread rapidly within prisons and into the community, and the high rates of IDU and other bloodborne virus risk behaviours among prisoners, there are logical benefits to ensuring that prisoners have high rates of immunity to infectious diseases. As such, we recommend a cost-benefit and feasibility analysis of implementing routine vaccination for measles, mumps, rubella and varicella, and exploration of options for improving uptake of HBV vaccination, such as accelerated schedules.

Acknowledgements: The VPDS was funded by the Justice Health and Forensic Mental Health Network. We thank the staff of the Population Health stream of Justice Health for conducting the VPDS and for providing additional information to help with preparation of this manuscript; the Institute of Clinical Pathology and Medical Research for serological testing of VPDS samples; and the National Centre for Immunisation Research and Surveillance for providing the Australian National Serosurveillance Program data.

Competing interests: All authors are current or former employees of Justice Health and Forensic Mental Health Network, which funded the study.

\section{Received 16 Jul 2012, accepted 29 Oct 2012}

1 Bick JA. Infection control in jails and prisons. Clin Infect Dis 2007: 45: 1047-1055.

2 Walkty A, Van Caeseele P, Hilderman T, et al. Mumps in prison: description of an outbreak in Manitoba, Canada. Can J Public Health 2011; 102: 341-344.
3 Valdarchi C, Farchi F, Dorrucci M, et al. Epidemiological investigation of a varicella outbreak in an Italian prison. Scand J Infect Dis 2008; 40: 943-945.

4 Levy MH, Quilty S, Young LC, et al. Pox in the docks: varicella outbreak in an Australian prison system. Public Health 2003; 117: 446-451.

5 Hutchinson SJ, Goldberg DJ, Gore SM, et al. Hepatitis B outbreak at Glenochil prison during January to June 1993. Epidemiol Infect 1998; 121: 185-191.

6 Khan AJ, Simard EP, Bower WA, et al. Ongoing transmission of hepatitis $B$ virus infection among inmates at a state correctional facility. Am J Public Health 2005: 95: 1793-1799.

7 Justice Health and Forensic Mental Health Network. Immunisation of patients. Sydney: Justice Health and Forensic Mental Health Network, 2010.

8 Butler T, Lim D, Callander D. National prison entrants' bloodborne virus and risk behaviour survey 2004, 2007, and 2010. Sydney: Kirby Institute, University of New South Wales \& National Drug Research Institute, Curtin University, 2011.

9 Gidding H. Australia's national serosurveillance program. N S W Public Health Bull 2003; 14 : 90-93.

10 Jardine A, Deeks SL, Patel MS, et al. An evaluation of the Australian National Serosurveillance Program. Commun Dis Intell 2010; 34: 29-36.

11 van Boven M, Kretzschmar M, Wallinga J, et al. Estimation of measles vaccine efficacy and critical vaccination coverage in a highly vaccinated population. JR Soc Interface 2010; 7 : 1537-1544.

12 Sutton AJ, Gay NJ, Edmunds WJ, et al. Modelling the hepatitis $B$ vaccination programme in prisons. Epidemiol Infect 2006; 134: 231-242.

13 Sutton AJ, Gay NJ, Edmunds WJ, Gill ON. Modelling alternative strategies for delivering hepatitis $B$ vaccine in prisons: the impact on the vaccination coverage of the injecting drug user population. Epidemiol Infect 2008; 136 1644-1649.

14 Gilbert RL, Costella A, Piper M, Gill ON. Increasing hepatitis $B$ vaccine coverage in prisons in England and Wales. Commun Dis Public Health 2004; 7 : 306-311.

15 Corben S. NSW inmate census 2010: summary of characteristics. Sydney: Corrective Services NSW, 2010. http://www.correctiveservices.nsw.gov.au/ data/assets/pdf_file/0006/293370/

statistical-publication-nsw-inmate-census2010.pdf (accessed Nov 2012).

16 Australian Government Department of Health and Ageing. The Australian Immunisation Handbook. 9th ed. Canberra: Department of Health and Ageing 2008.

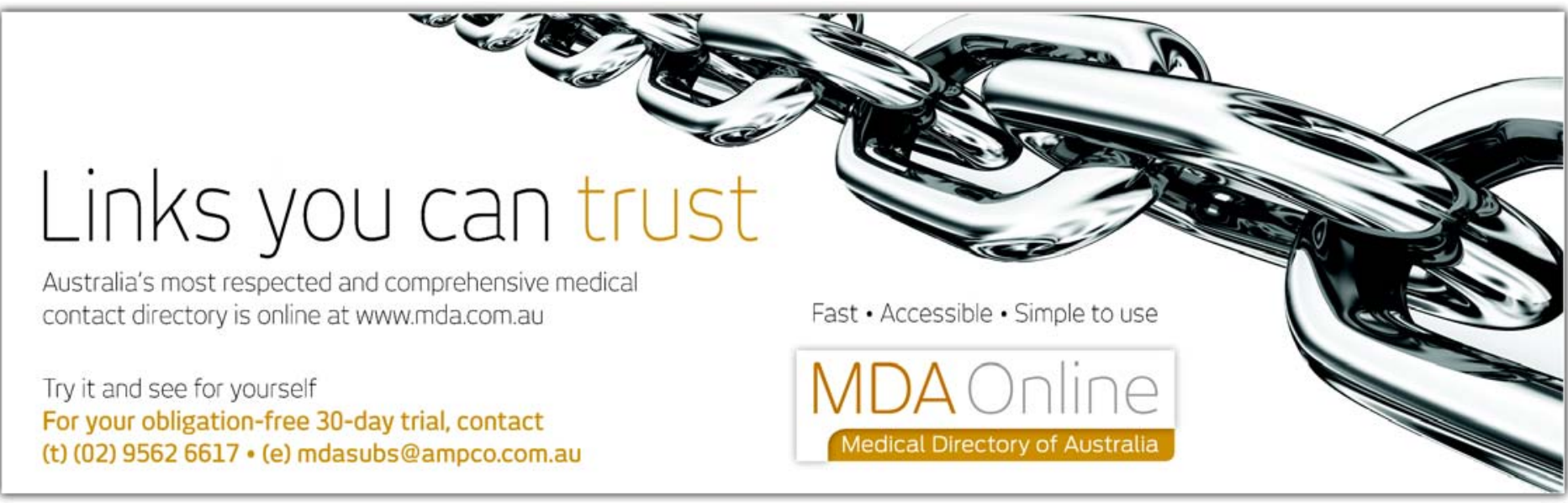

